Apidologie, 1972, 3 (3), 247-261.

\title{
ZUM PH-OPTIMUM DER SACCHARASE- UND MALTASEAKTIVITÄT DES PHARYNXDRÜSENSEKRETS DER BIENENARBEITERIN
}

\author{
Sur le pH optimum de l'activité de la saccharase et de la maltase \\ dans la sécrétion des glandes pharyngiennes de l'abeille ouvrière.
}

\author{
Klaus HALBERSTADT \\ Landesanstalt für Bienenkunde der \\ Universität Hohenheim
}

\begin{abstract}
SUMMARY
CONTRIBUTION TO THE PH-OPTIMUM OF THE SACCHARASE

AND MALTASE ACTIVITY OF THE PHARYNGEAL GLAND's

SECRETION OF WORKER BEES
\end{abstract}

Based on the pH-dependence of the enzymatic hydrolysis of sucrose and maltose by the worker bee's pharyngeal glands it is supposed that not one only but divers $\alpha$-glucosidases are produced. Especially in young bees the course of the pH-curves shows several maxima. Young bees seem to secrete an enzyme with greater affinity to maltose than to sucrose. The course of the curves also shows that caged bees produce another secretion than bees of the same age in colonies.

\section{ZUSAMMENFASSUNG}

Die pH-Abhängigkeit der enzymatischen Saccharose- und Maltosespaltung durch das Sekret der Pharynxdrüse der Bienenarbeiterin läßt vermuten, daß nicht nur eine, sondern verschiedene $\alpha$-Glucosidasen gebildet werden. Der Verlauf der pH-Kurven weist besonders bei jungen Bienen mehrere Maxima auf. Junge Bienen scheinen ein Enzym zu sezernieren, das eine größere Affinität zu Maltose besitzt als zu Saccharose. Bienen in Käfigen produzieren, wie aus dem Kurvenverlauf geschlossen werden kann, ein anderes Sekret als gleichaltrige Bienen aus einem Bienenvolk. 


\section{EINLEITUNG}

Die Verdauungsfermente der erwachsenen Honigbiene sind unter dem Gesichtspunkt ihrer biologischen Bedeutung schon öfter Gegenstand von Untersuchungen gewesen. Über ihre speziellen enzymologischen Eigenschaften besitzen wir dagegen nur wenige Kenntnisse. Das gilt besonders auch für die Enzyme der Pharynxdrüse. Im Honig wie in Versuchen mit Drüsenhomogenisaten gefundene Di- und Oligosaccharide (Srddiqui, 1970; Maurizio, 1957) erlauben es, die Pharynxdrüseninvertase als Glucosyltransferase bzw. Glucosaccharase zu klassifizieren (CAputto et al., 1966; Gottschalk, 1958). Aus diesen Befunden geht auch das Spektrum der möglichen Substrate dieses Enzyms hervor. Daten zur Reaktionskinetik sind bisher nur in Bezug auf die pH-Abhängigkeit der Saccharosespaltung gesammelt worden (NELson, 1924; GoNTarski, 1952). Nach diesen Untersuchungen besteht ein Optimalbereich für die enzymatische Hydrolyse der Saccharose ungefähr zwischen pH 5,5 und pH 6,3. Da die dort mitgeteilten Kurven einen sehr flachen Verlauf haben, läßt sich das pH-Optimum nicht enger fassen. Bei beiden Bearbeitungen ist das Alter der Versuchstiere nicht berücksichtigt worden. Es besteht darüberhinaus eine Abhängigkeit der Enzymproteine vom Genotypus : Das pHOptimum der Saccharosehydrolyse ist in gewisser Weise rassespezifisch (Gontarski, 1953). Weitere Kenntnis des Enzyms ist Untersuchungen der $\alpha$ Glucosidasefraktion der Honigproteine zu entnehmen (WhITE und KuSHNIR, 1967). Honigproteine tierischer Herkunft, die Saccharase- und Maltaseaktivität aufweisen, lassen sich chromatographisch und elektrophoretisch in ein Muster von 7 bis 18 Isoenzymen aufspalten. Die Zusammensetzung auch dieses Musters ist genetisch beeinflußt. Derartige Enzymmuster wurden bei vielen Insekten nachgewiesen (Latner und Skillen, 1968).

Das Ziel der vorliegenden Arbeit war es, zunächst die älteren Befunde über das pH-Optimum der Disaccharidaseaktivität des Pharynxdrüsensekrets mit modernen, enzymatischen Methoden nachzuprüfen und ferner, diese Untersuchungen auf die bisher vernachlässigten jungen Stockbienen auszudehnen. Da diese Altersgruppe ein von dem der Sammelbienen abweichendes, elektrophoretisches Proteinbild des Pharynxdrüsensekrets besitzt (HalberSTADT, 1966) ist wahrscheinlich, daß auch andere Enzymproteine vorliegen. Letzteres sollte sich in der $\mathrm{pH}$-Abhängigkeit der Geschwindigkeit des Substratabbaus äußern. Indem es möglich ist, daß Isoenzyme verschiedene $\mathrm{pH}$ Optima haben (Greenberg, 1964), kann unter geeigneten Versuchsbedingungen einem elektrophoretischen Muster, das Isoenzyme anthält, eine mehrgipfelige pH-Kurve entsprechen.

Die Auswahl der Substrate bei dieser Untersuchung beschränkte sich auf Saccharose und Maltose. Beide Zucker wurden mehrfach in Bearbeitungen 
des Pharynxdrüsensekrets einbezogen (Örösi PaL, 1968) und bieten daher die besten Vergleichsmöglichkeiten.

\section{METHODE}

\section{1. - Tiermaterial}

Ausgangsmaterial für die Untersuchungen bildeten Ableger von Wirtschaftsvölkern (Krainer Rasse). Untersucht wurden vor dem Flugloch abgefangene ältere Sammelbienen, Stockbienen verschiedenen Alters und im Käfig ohne Brut gehaltene Arbeiterinnen. Stockbienen und gekäfigte Bienen schlüpften im Brutschrank $\left(35^{\circ} \mathrm{C}\right)$. Stockbienen wurden in größeren Serien nach der Methode von Mc Donald und Levin (1965) mit Farbe markiert und in das Ursprungsvolk zurückgebracht. Eine Entnahme zur Präparation erfolgte in Abständen von 24 Stunden. Untersucht wurden Stockbienen bis zu einem Alter von 7 Tagen nach dem Schlüpfen. Da die Versuche während der Hauptwachstumsperiode der Völker durchgeführt wurden, hatten die Stockbienen im Versuchszeitraum den Höhepunkt der Entwicklung ihrer Futtersaftdrüsen teilweise bereits überschritten. Käfigbienen wurden ohne Königin oder Brut in Gruppen von 100 Tieren in Liebefelder Kästchen bis zu einem Alter von 10 Tagen gehalten. Als Nahrung diente ein Gemisch von verdünntem Honig mit Pollen ( $v: v=3$ : 1 , ad libitum).

\section{2. - Präparationstechnik}

Zur Präparation der Futtersaftdrüsen wurden die Versuchstiere mit Essigsäure-Äthylester abgetötet. Die herauspräparierten Drüsen wurden in eisgekühltem $\mathrm{H}_{2} \mathrm{O}$ aufgefangen, mit einem Homogenisator nach Potter-Elvehjem zerkleinert und einer Ultrafiltration unterworfen. Hierzu diente eine $5 \mathrm{ml}$ - Injektionsspritze (Luer-Lok - Ansatz) mit aufgesetzter Swinnex - 25 - Filtereinheit, bestückt mit Celluloseesterfilter SM (Porenweite $5 \mu \mathrm{m}$ ) und Glasfaser - Vorfilter AP 25 (Millipore GmbH, Neu Isenburg). Das gewonnene Filtrat wurde gefriergetrocknet (Gerät Texvac I, Textor, Bad Soden). Die getrockneten Präparate ließen sich, luftdicht verschlossen, bei $-15^{\circ} \mathrm{C}$ ohne Verlust an Aktivität über ein Jahr aufbewahren. Jedes Präparat enthielt die Sekretproteine von 20 Pharynxdrüsen (= 20 Individuen).

Zur enzymatischen Untersuchung ließen sich die Lyophilisate leicht in eisgekühltem 0,1 m wäss. NaCL lösen. Gelöste Präparate behielten in der Kälte ihre Enzymaktivität für mehrere Stunden.

Abgefangene Sammelbienen wurden nur dann weiterverarbeitet, wenn ibre Futtersaftdrüsen bereits in Degeneration begriffen waren. Die Drüsen datierter Stockbienen waren ab dem 3. Tag nach dem Schlüpfen voll entwickelt. In Gefangenschaft gahaltene Bienenarbeiterinnen zeigten ungleichmäßige Entwicklung ihrer Pharynxdrüsen.

\section{3. - Bestimmung der Enzymaktivität}

Die Ermittlung der Aktivität des Drüsenenzyms erfolgte an Hand der gebildeten Glucose. Es wurden zwei Methoden eingesetzt : 1948).

a) Manometrische Methode (Glucoseoxydase-Katalase-Methode; KeILIN und HaRTrEe,

Die Messung der Enzymaktivität bei Sammelbienen konnte mit üblichen DoppelkapillarManometern (Warburggerät VT 146, B. Braun, Melsungen) durchgeführt werden. Pro Ansatz für ein $13 \mathrm{ml}$ - Standardgefäß genügte die 1 Futtersaftdrüse entsprechende Menge gefriergetrockneten Materials. Diese Menge wurde bei stark degenerierten Drüsen verdoppelt. Zur Untersuchung von jungen Bienenarbeiterinnen mußten sog. VP - Mikromanometer mit $4 \mathrm{ml}$ - Mikrogefäßen eingesetzt werden, da hier die Enzymaktivität sehr niedrig ist. Eine zunächst versuchte Erhöhung der Enzymkonzentration im Standardgefäß führte zu einem schnellen Druckabfall, der möglicherweise auf der Reaktion der bei Jungbienen vorherrschenden Futtersaftproteine mit der Katalase beruht (SIZER, 1953). Im Mikrosystem konnte ein störungsfreier Ansatz bei einer Enzymkonzentration entsprechend 0,5 bis 1 Pharynxdrüse pro Manometer 
durchgeführt werden. Die Beschickung der Manometergefäße ist aus Tabelle 1 zu entnehmen. Das Gasvolumen bestand aus atmosphärischer Luft. Die Nachweisenzyme stammen von Boehringer GmbH (Mannheim).

TABELLE 1

\begin{tabular}{|c|c|c|}
\hline & Makrosystem & Mikrosystem \\
\hline $\begin{array}{l}\text { Präparat gelöst in } \\
0,1 \mathrm{~m} \text { wäss. NaCL }\end{array}$ & $1000 \mu l$ & $300 \mu l$ \\
\hline Puffer nach Me Ilvain & $1000 \mu \mathrm{l}$ & $300 \mu l$ \\
\hline Äthanol & $100 \mu \mathrm{l}(20 \%)$ & $25 \mu l(24 \%)$ \\
\hline Katalase & $100 \mu \mathrm{l}$ (enthaltend $0,4 \mathrm{mg}$ & $25 \mu \mathrm{l}$ (enthaltend $0,13 \mathrm{mg}$ \\
\hline Glucoseoxydase & $300 \mu 1$ (enthaltend $0,33 \mathrm{mg}$ & $100 \mu 1$ (enthaltend $0,11 \mathrm{mg}$ \\
\hline Substrat & $200 \mu \mathrm{l}$ (im Seitengefäß) & $50 \mu l($ im Seitengefä $\Omega$ ) \\
\hline KOH, $10 \%$ Filterpapier & $200 \mu \mathrm{l}$ (im Einsatzgefäß) & $50 \mu l($ im Einsatzgefäß) \\
\hline Flüssigkeitsvolumen $V_{t}$ & $3000 \mu l$ & $850 \mu l$ \\
\hline
\end{tabular}

Die Inkubation erfolgte bei $35^{\circ} \mathrm{C}$, die Meßrate betrug $40 \mathrm{~min}$ (4 Ablesungen). Die getesteten Substrate (Saccharose, Maltose) wurden in einer Konzentration von 3,4. 10-2 Mol vorgelegt. In einem Versuch mit Sammelbienen betrug die Konzentration des Substrats $1,6.10^{-3}$ Mol. Als Puffergemisch diente Natriumphosphat-Zitrat-Puffer nach Mc Ilvain (RAUEN, 1964), Endkonzentration $=0,1 \mathrm{~m}$. Die pH-Skala wurde in $0,2 \mathrm{pH}$ eingeteilt. Der untersuchte Bereich erstreckte sich von $\mathrm{pH} 4,6$ bis $\mathrm{pH} 7,2$. Das bei einem Versuch verwendete Drüsenmaterial entstammte jeweils einem gefriergetrockneten Präparat.

b) Photometrische Methode (Hexokinase - Glucose-6-phosphat - Dehydrogenase - Methode, BERGMEYER et al., 1970)

Zur Aktivitätsbestimmung wurden pro Ansatz $20 \mu \mathrm{l}$ in $0,1 \mathrm{~m}$ wäss. NaCL gelöstes Drüsenmaterial mit $20 \mu \mathrm{l}$ Puffer und $20 \mu \mathrm{l}$ Substratlösung bei $35^{\circ} \mathrm{C} 30 \mathrm{~min}$ inkubiert. Die Denaturierung der Drüsenenzyme nach Ablauf der Inkubationszeit erfolgte bei einem Teil der Versuche mit $500 \mu \mathrm{l}$ Uranylacetat $\left(0,16 \%\right.$ ), bei einem anderen Teil durch Hitze (Wasserbad, $90^{\circ} \mathrm{C}, 30$ sec) mit nachfolgender kurzer Zentrifugation. Die Enzymkonzentration pro Ansatz entsprach bei jungen Stockbienen einem Äquivalent von 0,156 Futtersaftdrüsen in $60 \mu \mathrm{l}$, bei Sammelbienen einem Äquivalent von 0,07 Futtersaftdrüsen in $60 \mu \mathrm{l}$. Entsprechend wurden die Verdünnungen des gefriergetrockneten Materials gewählt. Als Puffergemisch diente in einleitenden Versuchen Phosphat-Zitrat-Puffer nach Mc Ilvain (RAuEN, 1964), Endkonzentration $=0,1 \mathrm{~m}$, in allen weiteren Versuchen Veronal- Azetat-Puffer nach Michaelis (KLeinzeller, 1965, Endkonzentration $=0,003 \mathrm{~m}$. Die geringere Ionenstärke des zweiten Puffers erwies sich als vorteilhaft. Mc Ilvain-Puffer erlaubt nicht die Verwendung von Uranylazetat (WeNzL, 1966). Die Einteilung der pH-Skala erfolgte in Übereinstimmung mit der manometrischen Methode. Der untersuchte Bereich erstreckte sich von $\mathrm{pH} 4,0$ bis $\mathrm{pH} 7,6$. Die Substratkonzentration (Saccharose, Maltose) betrug 7,16. 10 $0^{-2}$ Mol. Die enzymatische Bestimmung der Glucose wurde mit dem Glucose-UV-Test (Boehringer GmbH, Mannheim) ausgeführt.

Die vom Hersteller vorgeschriebenen Testansätze mußten entsprechend dem geringen Inkubationsvolumen auf $1 / 2$ verkleinert werden. Die Extinktionsmessung wurde bei $340 \mathrm{~nm}$ vorgenommen (Selbstregistrierendes Spektralphotometer RPQ 20 A, Zeiss, Oberkochen).

\section{4. - Berechung der Enzymeinheiten}

Die Enzymaktvität ist als Volumenaktivität (BERgMEYer et al., 1970) ausgedrückt. Die Einheit $\mathrm{mU} / \mathrm{ml}$ wurde auf eine Pharynxdrüse (=1 Individuum) bezogen). Dadurch ist 
die einer Drüse entsprechende Enzymmenge eines gefriergetrockneten Präparates gelöst in einem einheitlichen Volumen ( $1 \mathrm{ml}$ ) wiedergegeben. Für die Berechnung der Volumenaktivität sei auf die zitierte Literatur verwiesen. Für die manometrische Methode kann die dort angegebene Formel infolge der Übereinstimmung von Inkubations- und Testvolumen in der vereinfachten Form $1000 . \Delta 0_{2} / \Delta t-1 / n$ Pharynxdrüse geschrieben werben. Die Volumenverhältnisse im Inkubationsansatz waren bei beiden Methoden gleich. Die Substratkonzentration lag in beiden Fällen über dem Sättigungswert, so daß die Reaktion mit maximaler Geschwindigkeit ablaufen konnte. Dadurch ist ein Vergleich beider Methoden möglich. Mit Maltose als Substrat gewonnene Meßwerte wurden halbiert, da nur eine Glucoseeinheit des Moleküls eine Reaktion mit dem Enzym eingeht.

Bezugseinheiten wie Frisch- oder Trockengewicht, Protein-oder DNS-Gehalt konnten nicht verwendet werden, da der Anteil an Fremdproteinen (Futtersaftproteinen) ebenso wie die DNS-Menge der Drüse starken altersmäßigen Schwankungen unterliegt (PAINTER und Biesele, 1966).

\section{5. - Vorversuche}

Durch Leerversuche wurde sichergestellt, daß die Drüsenpräparate kein natürliches Substrat für die zu messenden oder die eingesetzten Enzyme enthalten. Eine messbare Aktivität an natürlicher Glucoseoxydase konnte unter den Versuchsbedingungen (manometrische Versuche) nicht gefunden werden.

Da bei der manometrischen Methode die eingesetzten Nachweisenzyme mit dem Puffer des Ansatzes in Berührung kommen, war zu prüfen, wieweit das System Glucoseoxydase-Katalase pH-abhängig ist. Nach Vorlage von Glucose zeigten sich nur geringe Umsatzdifferenzen im Bereich der intersuchten pH-Skala (Abb. 1). In weiteren Vorversuchen wurde der Einfluß von Puffer, pH-Wert und Inaktivierungsmethode auf die nichtenzymatische Substratspaltung untersucht. Unter den beschriebenen Versuchsbedingungen treten derartige Fehler nicht auf. Die Empfindlichkeit der Saccharose gegenüber pH-Werten $<4,0$ gestattete allerdings nicht, die pH-Skala auf diesen Bereich auszudehnen.

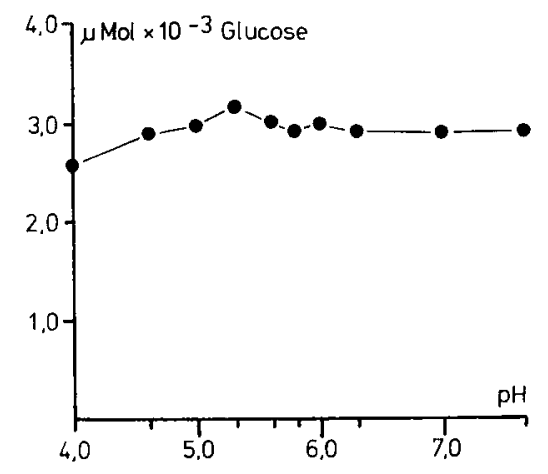

Авв. 1. - Einfluß der Pufferung des Ansatzes auf den manometrischen Glucosenachweis.

FIG. 1. - Influence du tamponnage de la prise d'essai sur la mise en évidence manométrique du glucose.

Die Reaktionsgeschwindigkeit war bei beiden Methoden linear abhängig von der Enzymkonzentration (Abb. 2). Eine gleichfalls lineare Abhängigkeit bestand zur Meßrate (Inkubationszeit). Die Reaktion mit Saccharose zeigte beim manometrischen Ansatz eine kurze Anlaufphase (Abb. 3). Eine Verfälschung der Ergebnisse durch Transferreaktionen des Drüse- 
nenzyms mit Reaktionsprodukten ist bei der manometrischen Methode durch die Weiterreaktion der Glucose nicht möglich. Bei der photometrischen Methode wurde die Inkubationszeit so kurz gewählt $(30 \mathrm{~min})$, daß der Fehler zu vernachlässigen sein dürfte.

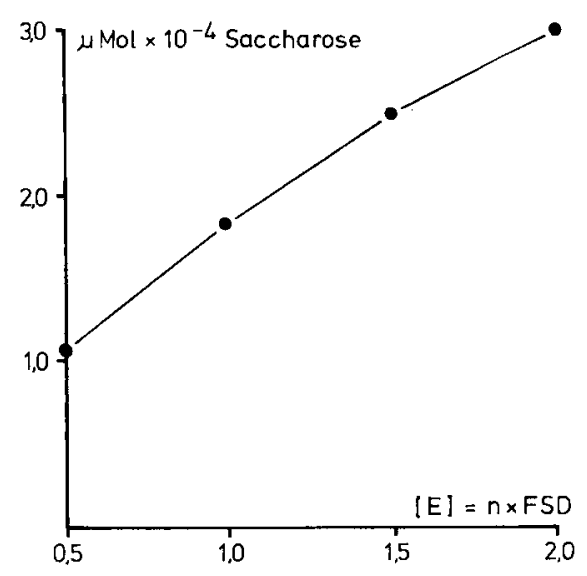

Авв. 2. - Beziehung zwischen der Enzymkonzentration und dem Saccharoseabbau. Enzymkonzentration [E] = Anzahl Futtersaftdrïsen.

Fig. 2. - Relation entre la concentration enzymatique et l'hydrolyse du saccharose. Concentration enzymatique $[\mathbf{E}]=$ nombre de glandes pharyngiennes.

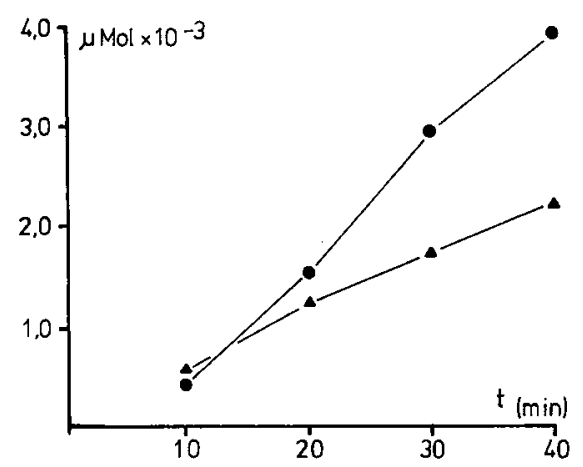

Авв. 3. - Einfluß der Inkubationszeit t auf die Menge der enzymatisch gespaltenen Saccharose $(\bullet-\bullet)$ und Maltose $(\mathbf{\Delta}-\mathbf{\Delta})$.

FIG. 3. - Influence du temps d'incubation t sur la quantité de saccharose hydrolysé par voie enzymatique $(\bullet-\bullet)$. Maltose : 


\section{ERGEBNISSE}

\section{1. - Alte Bienenarbeiterinnen (Sammelbienen)}

Das Pharynxdrüsensekret älterer Bienenarbeiterinnen besitzt einen hohen Enzymspiegel. Die Volumenaktivität beträgt bei den Versuchstieren unter den angegebenen Bedingungen für Saccharose als Substrat 6000 bis 18000 Einheiten, für Maltose 1000 bis 6000 Einheiten. Die Abhängigkeit des Substratverbrauchs vom $\mathrm{pH}$ des Ansatzes verlief bei 15 untersuchten Präparaten gleichsinnig. Die daraus resultierende Kurve ist unregelmässig (Abb. 4). Bei Vorlage von Saccharose ergeben sich 2 optimale Bereiche von $\mathrm{pH} 5,4$ bis pH 5,8 und pH 6,4 bis pH 6,6. Vorlage von Maltose liefert einen Kurvenverlauf, der nicht deutlich in 2 Optima unterteilt ist. Der Optimalbereich zwischen $\mathrm{pH} 5,8$ und $\mathrm{pH}$ 6,8 ist gegenüber der Saccharosekurve zum basischen hin verschoben. Das Maximum liegt bei $\mathrm{pH}$ 6,2. Die genannten Verhältnisse gelten für eine Substratkonzentration von 3,4. $10^{-2}$ Mol. Vorlage von Saccharose in einer Konzentration von 1,6.10 $10^{-3} \mathrm{Mol}$ führte zu einem anderen Kurvenverlauf : Ein Optimum im Bereich von $\mathrm{pH}$ 6,4 bis 6,6 ist nicht feststellbar.

Saccharose wird im Vergleich zu Maltose über die gesamte geprüfte $\mathrm{pH}$ Skala mit einer 2 bis 3 -fachen Geschwindigkeit gespalten (Abb. 4).

\section{2. - Junge Bienenarbeiterinnen (Stockbienen)}

Der Enzymspiegel des Pharynxdrüsensekrets von Stockbienen im Alter von 1 bis 7 Tagen beträgt bei den Versuchstieren im Durchschnitt $1 / 60$ der Enzymmenge der Sammelbienen. Bei Vorlage von Saccharose wird eine Volumenaktivität von 150 bis 400 Einheiten, bei Vorlage von Maltose eine Aktivität von 50 bis 350 Einheiten erreicht. Die Abhängigkeit des Substratverbrauchs vom $\mathrm{pH}$ des Ansatzes entspricht nicht der bei Sammelbienen. Es resultiert ein Kurvenverlauf, der mehrere Maxima aufweist. Das dominierende Optimum für die Saccharosespaltung erstreckt sich über den Bereich von pH 5,4 bis 5,8 (Abb. 5).

Nebenmaxima wurden in den pH-Bereichen 4,6 bis 4,$8 ; 5,3 ; 6,0$ bis 6,2 und 6,8 bis 7,0 gefunden (Abb. 6). Die enzymatische Spaltung der Maltose ist in anderer Weise vom $\mathrm{pH}$ abhängig. Der optimale Bereich liegt zwischen pH 5,8 und pH 7,4. Der Kurvenverlauf ist in Maxima bei pH 6,4;7,0 und 7,4 gegliedert. Ein weiteres Maximum findet sich im pH-Bereich 4,8 bis 5,2. Die Spaltung der Saccharose verläuft unterhalb von $\mathrm{pH} 5,6$ etwa 2 bis 3 -mal so schnell wie die Spaltung der Maltose. Oberhalb von $\mathrm{pH} 5,6$ verändert sich 


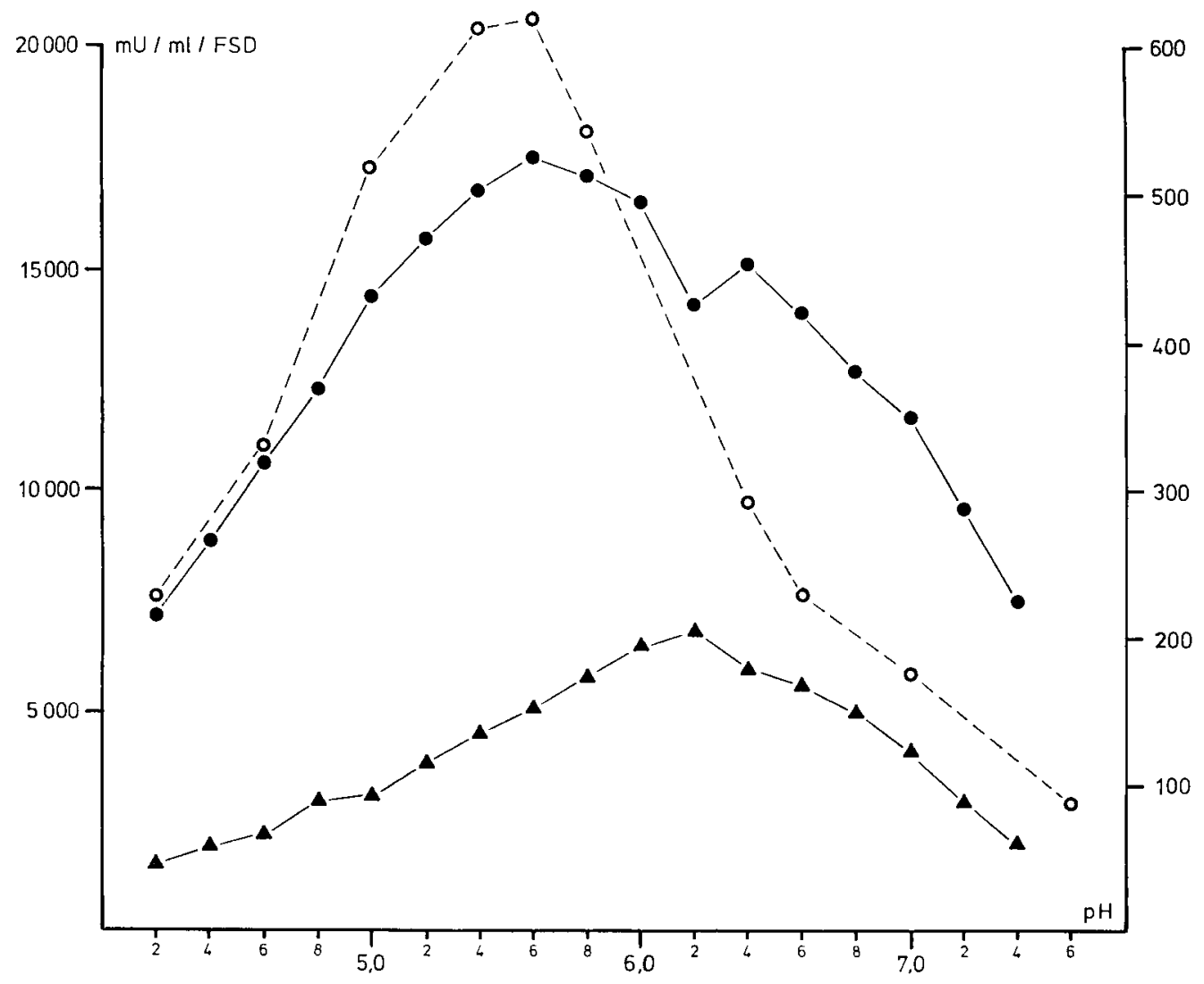

Авв. 4. - Abhängigkeit der Enzymaktivität $[\mathrm{mU} / \mathrm{ml} / \mathrm{FSD}]$ des Pharynxdrüsensekrets vom $\mathrm{pH}-\mathscr{W}$ ert des Ansatzes bei Flugbienen (= ältere Sammlerinnen). Linke Ordinate : Konzentration der Saccharose $(\bullet-\bullet)$ und Maltose $(\mathbf{A}-\mathbf{A})=3,4.10^{-2}$ Mol. Rechte Ordinate : Konzentration des Saccharose $(\mathrm{O}-\mathrm{O})=1,6.10^{-3} \mathrm{Mol}$. Mittelwerte aus 15 gefriergetrockneten Präparaten von je 20 Bienenarbeiterinnen.

FIc. 4. - Relation entre l'activité enzymatique (mU / ml / FSD) de la sếcrêtion des glandes pharyngiennes et le $\mathrm{pH}$ de la prise d'essai chez les butineuses âgées. Ordonnées de gauche : concentration du saccharose

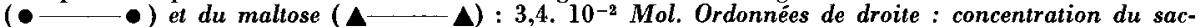
charose $(\mathrm{O}-\mathrm{O}): 1,6.10^{-2} \mathrm{Mol}$. Valeurs moyennes de 15 préparations lyophilisées de chacune 20 ouvrières. 


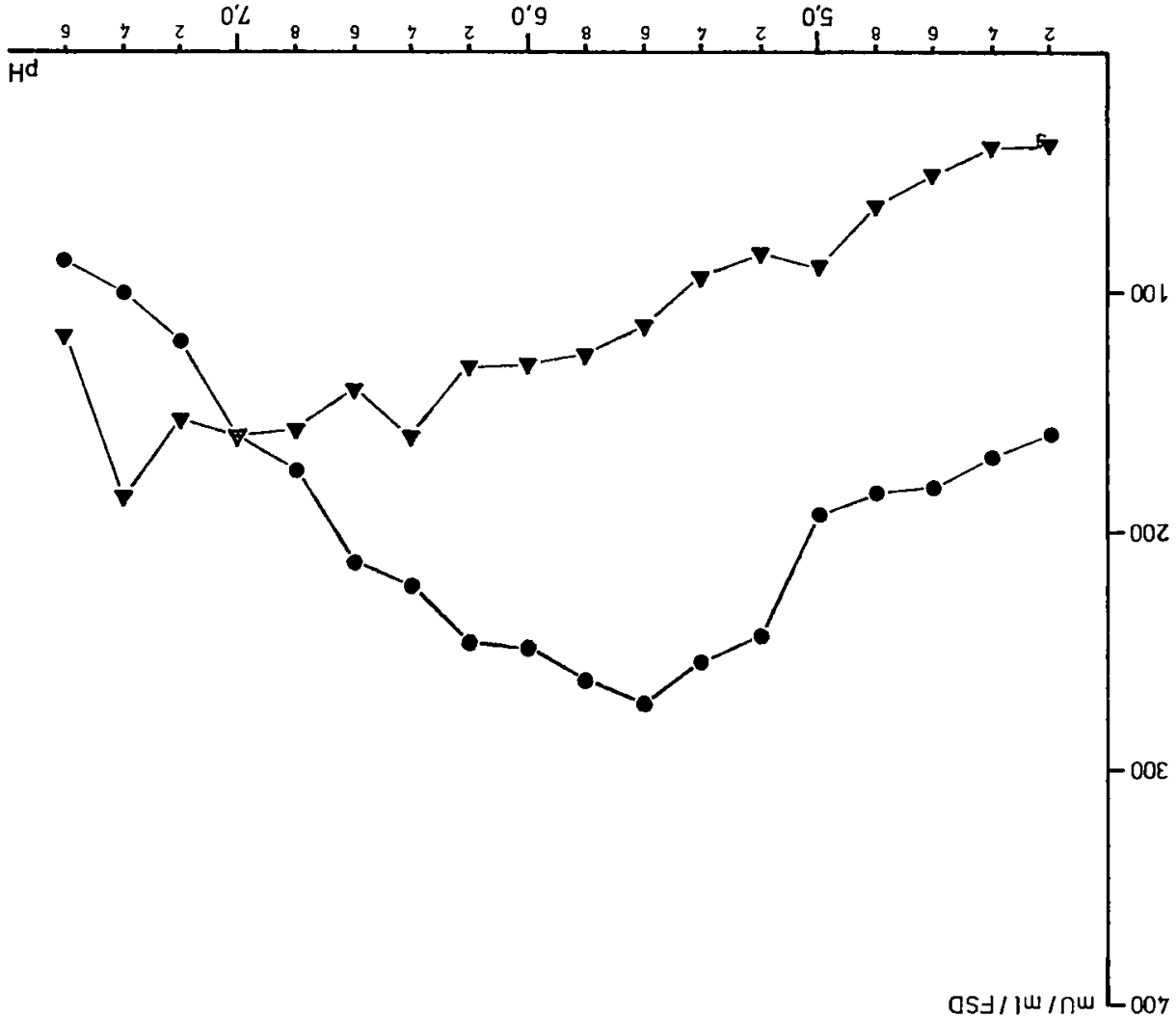

Aвв. 5. - Abhängigkeit der Enzymaktivität des Pharynxdrüsensekrets vom pH-Wert des Ansatzes bei Stockbienen (1-7 Tage alt). Konzentration der Saccharose (•-—) und Maltose (A $=7,16.10^{-2}$ Mol. Mittelwerte aus 30 gefriergetrockneten Präparaten von je 20 Bienenarbeiterinnen.

Fig. 5. - Relation entre l'activité enzymatique de la sécrétion des glandes pharyngiennes et le $\mathrm{pH}$ de la prise d'essai chez les abeilles jeunes (1 à 7 jours). Concentration du saccharose ( $-\bullet)$ et du maltose $(\mathbf{A}-\mathbf{A})=7,16.10^{-2} \mathrm{Mol}$. Valeurs moyennes de 30 prêparations lyophilisêes de chacune 20 ouvrières. 


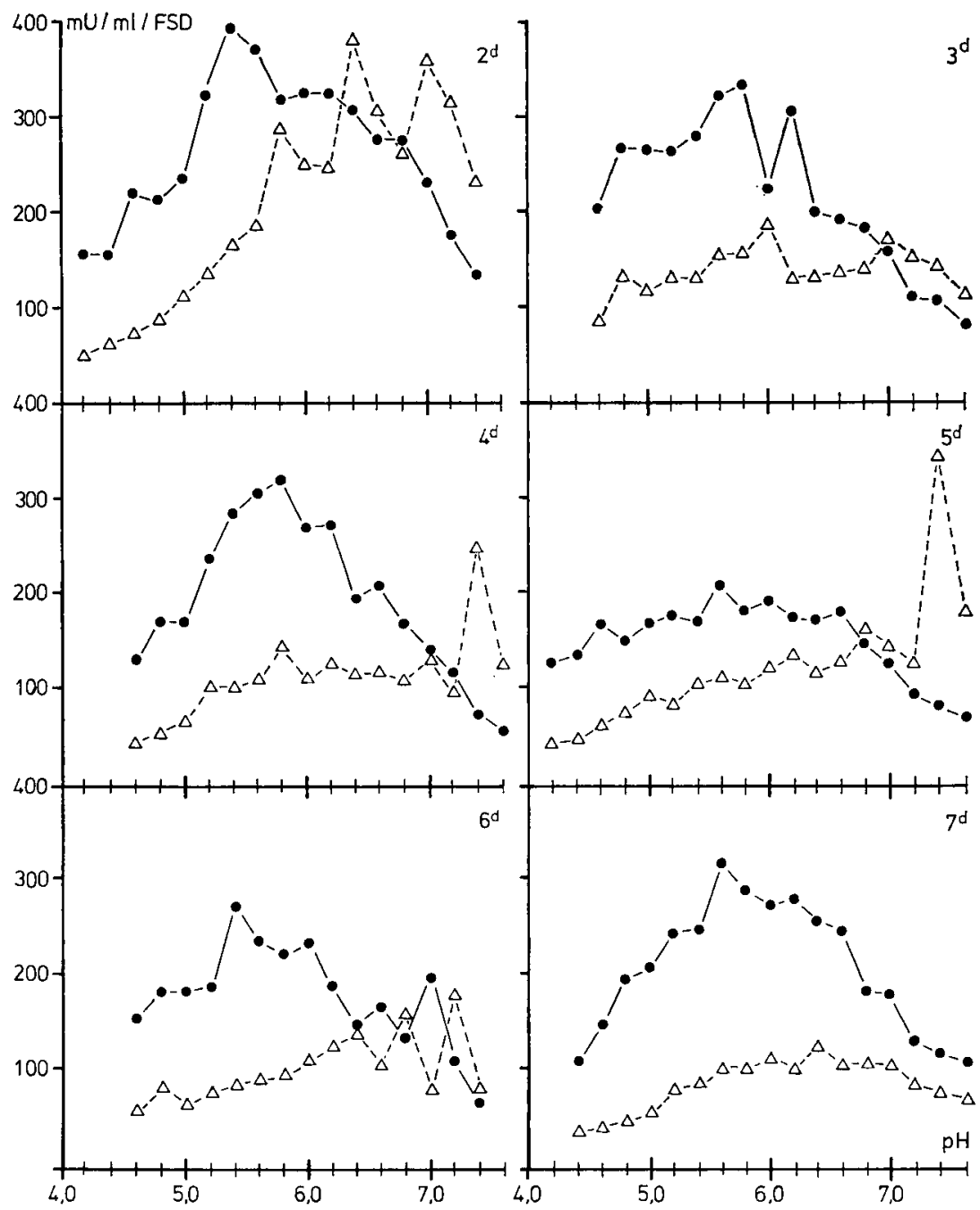

Aвв. 6. - Beispiele für die Variabilität in der Enzymaktivität einzelner gefriergetrockneter Präparate von Stockbienen. Bei 7 Tage alten Arbeiterinnen änelt der Kurvenverlauf in der Regel dem bei Flugbienen.

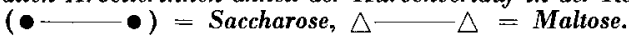

Fig. 6. - Exemples individuels de la variabilité de l'activité enzymatique de préparation lyophilisées d'abeilles jeunes. En règle générale à 7 jours la courbe ressemble à celle des butineuses. ( $\bullet-\bullet)$ : saccharose. $(\triangle \longrightarrow \triangle)$ : maltose. 
diese Beziehung langsam bis zu einer gleichen Reaktionsgeschwindigkeit mit beiden Substraten um den Neutralpunkt. Im basischen Teil der pH-Skala wird Saccharose langsamer als Maltose gespalten (Abb. 5). Diese Verhältnisse unterliegen je nach Präparat gewissen Schwankungen. Abb. 6 gibt Beispiele für den Kurvenverlauf bei einzelnen Versuchen. Abb. 5 ist aus den Durchschnittswerten aller Messungen (30 Präparate) gewonnen. Der Einfluss des Alters der Versuchstiere macht sich erst bei 7 Tage alten Arbeiterinnen bemerkbar. In diesem Stadium ist der Kurvenverlauf bei den meisten Proben ausgeglichener. Es besteht dann eine Ähnlichkeit zur pH-Abhängigkeit der Hydrolyse der getesteten Substrate bei Sammelbienen (Abb. 4).

\section{3. - In Gefangenschaft gehaltene Bienenarbeiterinnen}

Bei im Käfig gehaltenen Arbeiterinnen konnte eine Veränderung der pH-Abhängigkeit der Disaccharidspaltung bis zum 10. Tag nach dem Schlüpfen beobachtet werden. Die von den Versuchstieren sezernierte Enzymmenge ist zu Anfang geringer als bei gleichaltrigen Stockbienen. Sie erreicht nach 5 Tagen aber bereits $1 / 10$ des Enzymspiegels bei Sammelbienen, d.h. 1000 bis 2000 Einheiten. Die Kurve des Substratverbrauchs in Abhängigkeit vom $\mathrm{pH}$ weist bei 2 Tage alten Arbeiterinnen 2 Maxima auf im Bereich von $\mathrm{pH} 5,4$ und pH 5,8 bis pH 6,0. 5 Tage alte Bienenarbeiterinnen zeigen ähnliche Verhältnisse. Bei 10-tägigen Arbeiterinnen konnte nur noch ein optimaler Bereich festgestellt werden zwischen $\mathrm{pH} \mathrm{5,4}$ und $\mathrm{pH} \mathrm{6,0} \mathrm{(Abb.} \mathrm{7).} \mathrm{Beide} \mathrm{getestete}$ Substrate verhielten sich gleich. Es wurden 20 Präparate untersucht.

\section{DISKUSSION}

Die graphische Darstellung der Enzymaktivität in Abhängigkeit vom pH-Wert der Reaktion liefert beim Vorliegen nur eines Ferments Kurven mit einem Maximum und einem mehr oder minder gleichmäßigen Abfall nach den extremen pH-Werten. Solche Verhältnisse konnten bei der Spaltung von Saccharose und Maltose durch das Pharynxdrüsensekret der Honigbiene nicht gefunden werden. Der Verlauf der pH-Kurven ist vielmehr in der Mehrzahl der Fälle unregelmäßig, d.h. es besteht ein optimaler Bereich, der von Nebenmaxima umgeben ist. Ein ähnlicher Kurvenverlauf wurde für die $\alpha, \alpha$ - Trehalaseaktivität im Mitteldarm von Calliphora erythrocephala ermittelt (WenzL, 1966). Die Ursache dafür dürfte im Vorhandensein von mehreren Enzymen mit überlappender Substratspezifität oder aber in einer Reihe von Isoenzymen gleicher Spezifität liegen. Bei dem Pharynxdrüsensekret sind vielleicht einige Isoenzyme beteiligt. Dafür spricht der relativ ähnliche Kur- 


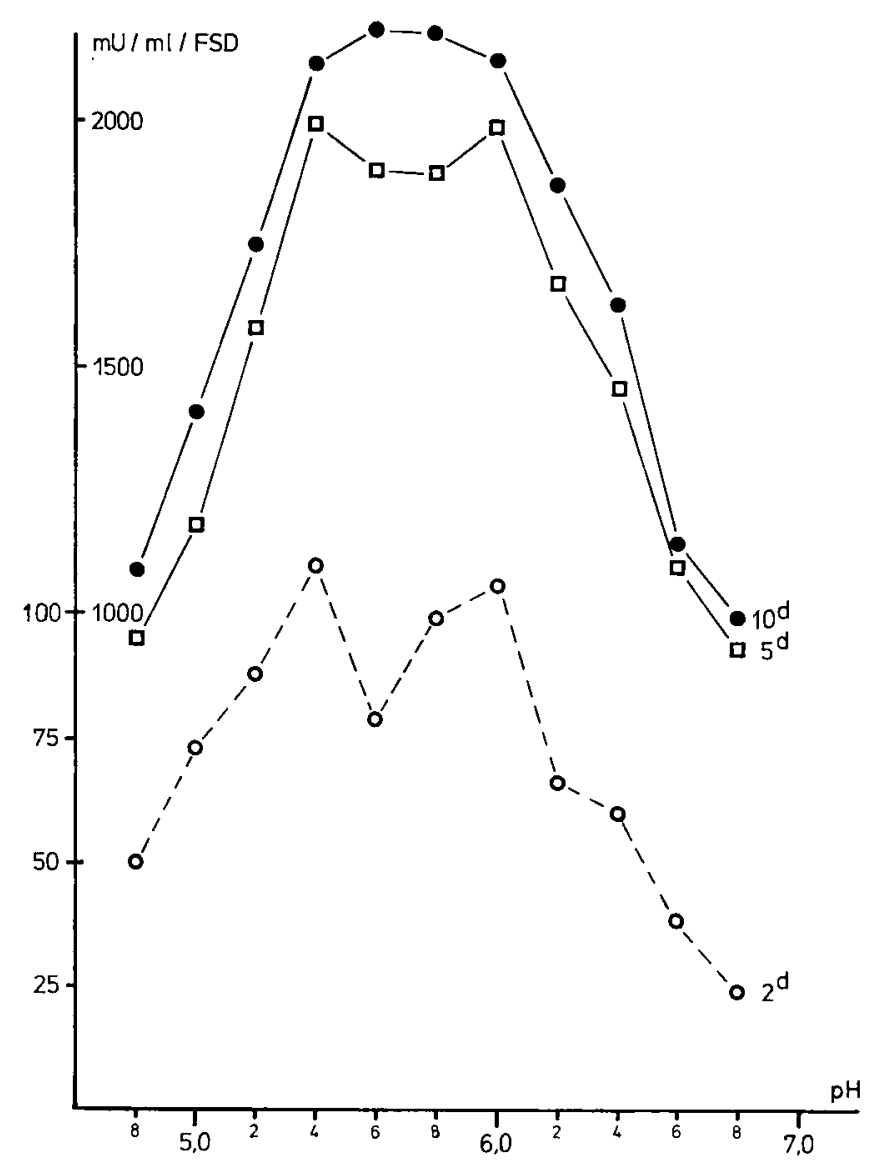

Aвв. 7. - Abhängigkeit der Enzymaktivität des Pharynxdrüsensekrets vom $\mathrm{pH}-W$ ert des Ansatzes bei gefangen gehaltenen Bienen. Saccharose, 3,4.10 $10^{-2} \mathrm{Mol}$. 2 Tage $=$ Ordinate links, 5 und 10 Tage $=$ Ordinate rechts.

Mittelwerte aus 20 gefriergetrockneten Präparaten von je 20 Bienenarbeiterinnen.

FIG. 7. - Relation entre l'activitê enzymatique de la sécrétion des glandes pharyngiennes et le $\mathrm{pH}$ de la prise d'essai chez les abeilles encagées. Saccharose, 3,4. $10^{-2} \mathrm{Mol}$. 2 jours : ordonnées de gauche 5 à 10 jours : ordonnées de droite.

Valeurs moyennes de 20 préparations lyophilisées de chacune 20 ouvrières.

venverlauf beider Substrate bei Jungbienen im sauren und bei Altbienen im gesamten pH-Bereich und auch die gleichbleibende Relation von Saccharoseabbau zu Maltoseabbau (= $3: 1)$ in den genannten Abschnitten der pH-Skala. WITE und Kushnir (1967) vermuten, daß die von ihnen aus Honig isolierten Glucosaccharasen tierischer Herkunft Isoenzyme sind. Sie konnten nachweisen, daß die enzymatische Aktivität gegenüber Saccharose und Maltose auf die gleichen Proteinfraktionen zurückgeht, wobei ebenfalls ein Verhältnis von $3: 1$ in der Hydrolysegeschwindigkeit von Saccharose und Maltose bestand. 
Die gleiche Affinität zu den beiden Substraten wurde auch bei Maltasen aus dem Darm von Wirbeltieren gefunden (DAhlquist, 1959). Sie scheint ein Charakteristikum der Glucosaccharasen zu sein.

Im Gegensatz dazu beobachtet man bei Jungbienen eine Verschiebung des Verhältnisses der Hydrolysegeschwindigkeit zugunsten der Maltose im basischen Abschnitt der pH-Skala. Es ist denkbar daß diese Enzymaktivität, deren Optimum bei pH 7,4 liegt, auf einem weiteren, abweichenden Enzym beruht. Dieses Ferment scheinen nur die jungen Bienenarbeiterinnen zu haben und seine Bildung wird womöglich mit der am Ende der Ammenbienenphase erfolgenden Umstellung in der Drüsensekretion (HaLberstadT, 1970) eingestellt.

Es ist auffällig, daß das Sekret von jungen Stockbienen einen komplizierteren Kurvenverlauf liefert als das von Flugbienen. Diese Erscheinung ist eine Parallele zu den elektrophoretischen Proteinmustern des Drüsensekrets beider Stadien; junge Bienenarbeiterinnen sezernieren eine Vielzahl von Eiweißen von im einzelnen geringer Menge, ältere einige wenige Proteine in hoher Quantität (HALberstadt, 1966). Damit kann allerdings noch nicht gesagt sein, daß Flugbienen nur so viele Glucosaccharasen bilden, wie Maxima in der pH-Kurve auftauchen. Abgesehen von der Unmöglichkeit, mit der Methode die genaue Zahl der Isoenzyme festzustellen, muß damit gerechnet werden, daß schwächere Aktivitäten von quantitativ bedeutenderen Enzymfraktionen überdeckt werden. Letzteres kommt vermutlich auch in den Kurven zum Ausdruck, die man erhält, wenn man eine geringe, nicht optimale Substratkonzentration einsetzt (Abb. 4).

Ohne Brut und Königin im Käfig gehaltene junge Bienen scheinen einer Umwelt ausgesetzt zu sein, die eine, unter Umständen tiefgreifende physiologische Umstellung bei ihnen hervorruft. Dies kommt bereits im Proteinspektrum der Futtersaftdrüsen zum Ausdruck, das mit seiner geringen Anzahl von Fraktionen dem von Winterbienen ähnelt (Halberstadt, 1966). Vermutlich in Analogie dazu haben auch die ermittelten pH-Aktivitätskurven dieser Bienen einen einfacheren Verlauf als die von gleichaltrigen jungen Arbeiterinnen im Stock.

Die Ursache der im einzelnen noch nachzuweisenden Unterschiede in den Fermenten von jungen und alten Bienenarbeiterinnen liegt wohl in den unterschiedlichen Aufgaben beider Altersklassen. Vielleicht ist die Glucosidasenausstattung der jungen Stockbienen keine Vorstufe der späteren Enzymsekretion, sondern eine Anpassung an die Aufgaben der Brutamme. 


\section{RESUMÉ}

Les données fournies par la littérature sur les relations entre le pH et l'hydrolyse enzymatique des sucres par la sécrétion des glandes pharyngiennes de l'abeille ne sont pas claires. L'irrégularité des courbes qu'on y trouve permet de supposer que les résultats qui ont été obtenus ne reposent pas sur l'existence d'une enzyme unique. Dans le miel, l'existence de plusieurs protéines glucolytiques a été démontrée; d'après leur comportement électrophorétique il peut s'agir d'iso-enzymes.

Dans le présent travail on a tenté de déterminer au moyen de méthodes nouvelles le $\mathrm{pH}$ optimum pour l'hydrolyse du saccharose et du maltose par la sécrétion glandulaire. On a utilisé une méthode manométrique (méthode de la glucose-oxydase-catalase) et une méthode photométrique (méthode de l'hexokinase-glucose-6-phosphate-déhydrogénase). Les deux méthodes ont fourni des résultats comparables. Étant donné que, jusqu'ici, on n'a expérimenté, pour tenter de répondre à la question posée, que sur des abeilles d'âge quelconque, nous avons pensé préférable d'utiliser des abeilles d'âge connu. Nos recherches ont porté sur des ouvrières âgées (butineuses), sur des ouvrières jeunes (de 1 à 7 jours) et sur des ouvrières maintenues en cagette, sans reine et sans couvain et âgées de 1 à 10 jours. Nous avons été incités à faire des distinctions en fonction des classes d'âge imaginal par la connaissance que nous avons des différences qui existent entre abeilles jeunes et âgées dans le comportement électrophorétique des sécrétions pharyngiennes.

Les diagrammes montrent que, même avec des méthodes très sensibles, on ne trouve pas de pH optimum uniforme pour l'hydrolyse enzymatique du saccharose et du maltose. Chez les ouvrières âgées la courbe de l'hydrolyse du saccharose en fonction du $\mathrm{pH}$ présente deux maxima (pH $[5,4$ à 5,8 et $\mathrm{pH} 6,4$ à 6,6 ); la courbe d'hydrolyse du maltose présente un domaine de pH optimum allant de $\mathrm{pH} 5,8$ à pH 6,8 (Fig. 4). Chez les abeilles jeunes la courbe est sensiblement différente (Fig. 5). L'hydrolyse du saccharose présente un domaine de $\mathrm{pH}$ optimum allant de $\mathrm{pH} 5,4$ à $\mathrm{pH} 5,8$ et des maxima pour $\mathrm{pH} 4,6$ à 4,8, $\mathrm{pH} 5,8, \mathrm{pH} 6,0$ à 6,2 et $\mathrm{pH} 6,8$ à 7. L'hydrolyse du maltose en fonction du $\mathrm{pH}$ se fait différemment. Le domaine optimum est situé entre 5,8 et 7,4; des maxima existent pour $\mathrm{pH} 4,8$ à 5,2, $\mathrm{pH} 6,4$ et $\mathrm{pH}$ 7,0 à 7,4. L'activité enzymatique des glandes pharyngiennes des jeunes abeilles n'est que le $1 / 60$ de l'activité des glandes pharyngiennes des abeilles âgées. Le saccharose est toujours hydrolysé plus rapi dement que le maltose chez les abeilles âgées et uniquement dans le domaine acide de l'échelle des pH chez les abeilles jeunes (Fig. 5). Lorsque l'on maintient de jeunes ouvrières en cagette, sans reine et sans couvain, il semble que la synthèse des enzymes des glandes pharyngiennes se déroule autrement que chez les ouvrières d'une colonie normale. La courbe de l'hydrolyse des disaccharides en fonction du $\mathrm{pH}$ est plus simple. Les maxima se trouvent à $\mathrm{pH} 5,4$ et 5,8 jusqu'à 6,0 . Si les abeilles encagées ont 5 à 6 jours, la distinction des 2 optimums n'est plus possible (Fig. 7). La concentration des enzymes chez les jeunes abeilles encagées atteint $1 / 10$ de celle des abeilles âgées. Le fait que l'hydrolyse enzymatique des deux substrats présente une telle irrégularité conduit à faire l'hypothèse que l'abeille produit plusieurs glucosidases dans ses glandes pharyngiennes. Ceci serait surtout vrai pour les jeunes stades (abeilles d'intérieur jusqu'à la fin de la période où elles sont nourrices). Cette classe d'âge se différencie des ouvrières plus âgées avant tout par l'hydrolyse rapide du maltose à $\mathbf{p H} 7,4$.

Quelques résultats indiquent que la courbe des $\mathrm{pH}$ peut être influencée par la concentration du substrat (Fig. 4).

\section{LITERATUR}

Bergmeyer H. U., 1970. Methoden der enzymatischen Analyse. Verlag Chemie, Weinheim, Bd. 2, p. 1163. 
Caputto R., R. E. Trucco und P. B. McCay, 1966. Glycosyltransferases (Transglycosylases), In : K. Lavg, Hrsg., Handbuch der physiologisch und pathalogisch chemischen Analyse Springer-Verlag, Berlin, Bd. 6 : Enzyme, Teil B, p. 253.

Dahlquist A., 1959. Studies on the heat inactivation of intestinal invertase, maltase and trehalase. Acta chem. Scand., 13, 945-953.

GonTaRSKı H., 1952. Fermentbiologische Studien an Bienen. 1. Das physiko-chemische Verhalten der kohlenhydratspaltenden Fermente. a) Invertierende Enzyme. Verhandl. Dt. Ges. angew. Entomol., Frankfurt, 186-197.

Gontarski H., 1953. Über physiologische Unterschiede bei Bienen verschiedener Abstammung. Z. Bienenforsch., 2, 98-108.

GotrschalK A., 1958. The enzymes controlling hydrolytic, phosphorolytic and transfer reactions of the oligosaccharides. In : W. RuHLAND, Hrsg., Handbuch der P flanzenphysiologie. Springer-Verlag, Berlin, Bd. 6, p. 87.

Greenberg D. M., 1964. Amino acid metabolism. Ann. Rev. Biochem., 33, 633-666.

Halberstadt K., 1966. Über die Proteine der Hypopharynxdrüse der Bienenarbeiterin. 1. Elektrophoretischer Vergleich von Sommer-, Winter- und gekäfigten Bienen. Ann. Abeille 9, 153-163.

Halberstadt K., 1970. Ein Beitrag zur Ultrastruktur und zum Funktionszyklus der Pharynxdrüse der Honigbiene (Apis mellifica L.), Cytobiologie, 2, 341-358.

Haydak M. H., 1970. Honey bee nutrition. Ann. Rev. Entomol., 15, 143-156.

KeILin D. und E. F. Hartree, 1948. The use of glucose oxidase (notadin) for the determination of glucose in biological material and for the study of glucose - producing systems by manometric methods. Biochem. J., 42, 230-238.

KLeinzeller A., 1965. Manometrische Methoden und ihre Anwendung in der Biologie und Biochemie. Gustav Fischer Verlag, Jena, p. 267.

Latner A. L. und A. W. Skillen, 1968. Isoenzymes in biology and medicine, Academic Press, London, p. 128.

Maunizio A., 1957. Zuckerabbau unter der Einwirkung der invertierenden Fermente in Pharynxdrüsen und Mitteldarm der Honigbiene (Apis mellifica L.), l. Sommerbienen der Krainer- und nigra-Rasse. Insectes sociaux, 4, 225-243.

McDonald J. L. und M. D. Levin, 1965. An improved method for marking bees, J. Apic. Res., 4, 95-97.

Nesson J. M. und D. J. Cohn, 1924. Invertase in honey, J. Biol. Chem., 61, 193-224.

Örösi Pal Z., 1968. Physiologie des glandes nourricières. In : B. Chauvin, Hrsg., Traité de biologie de l'abeille. Verlag Masson et Cie, Paris, Bd. 1, p. 263.

Painter T. S. und J.-J. Biesele, 1966. The fine structure of the hypopharyngeal gland'cell of the honey bee during development and secretion, Proc. national Acad. Sci. USA, 5, 1414-14.19.

RaUen H., 1964. Biochemisches Taschenbuch. Springer-Verlag, Berlin, Bd. 2, p. 90.

Siddiqui J. R., 1970. The sugars of honey. Adv. Carbohydrate Chem. Biochem., 25, 285309.

Srzer I. W., 1953. Oxidation of Proteins by tyrosinase and peroxidase, Adv. Enzymol., 14, 129-161.

WenzL H., 1966. Verdauung und Verwertung von Kohlenhydraten durch Calliphora erythrocephala Meig. Dissertation, Würzburg.

White J. W. und I. Kushin, 1967. The enzymes of honey : Examination by ion-exchange chromatography, gel filtration, and starch-gel electrophoresis, J. Apic. Res., 6, 69-89.

Anschrift des Verfassers :

Dr. K. Halberstadt, Landesanstalt für Bienenkunde der Universität Hohenheim, 7 Stuttgart 70, Emil-Wolff-Straße 60. 